Revista Universo Contábil, ISSN 1809-3337

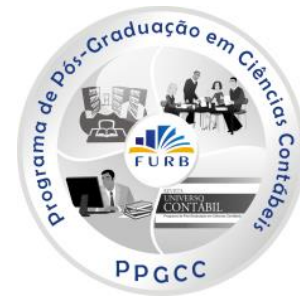

\title{
IDENTIFICAÇÃO DOS FATORES QUE INFLUENCIAM NA ESCOLHA DA FORMA DE INVESTIMENTO EM CRESCIMENTO (ORGÂNICO OU INORGÂNICO) DAS INDÚSTRIAS BRASILEIRAS 1
}

\section{IDENTIFICATION OF FACTORS THAT INFLUENCE THE CHOICE OF THE FORM OF INVESTMENT IN GROWTH (ORGANIC OR INORGANIC) OF BRAZILIAN INDUSTRIES}

\section{IDENTIFICACIÓN DE LOS FACTORES QUE INFLUYEN EN LA ELECCIÓN DE LA FORMA DE INVERSIÓN EN EL CRECIMIENTO (ORGÁNICO O INORGÁNICO) DE LAS INDUSTRIAS BRASILEÑAS}

\author{
João Francisco Morozini \\ Doutor em Administração de Empresas pela MACKENZIE/SP \\ Professor da Universidade Estadual UNICENTRO \\ Endereço: Rua Padre Salvador, 875 Bairro Santa Cruz \\ CEP: 85100-970 - Guarapuava - PR \\ E-mail: jmorozini@uol.com.br \\ Telefone: (42) 3621-1072 \\ Diógenes Manoel Leiva Martin \\ Doutor em Administração de Empresas pela FGV/SP \\ Professor do Doutorado em Administração de Empresas MACKENZIE/SP \\ Endereço: Rua da Consolação, 930, Bairro Consolação \\ CEP 01302-907- São Paulo - SP \\ E-mail: diomartin@mackenzie.br \\ Telefone: (11) 2114-8000
}

\section{RESUMO}

As teorias que tratam sobre a decisão de investimento da firma, sejam elas de caráter neoclássico ou não, tendem a considerar a influência das estruturas organizacionais na determinação do investimento. Nas Corporações modernas, para as estratégias empresariais e as estruturas organizacionais, são levados em consideração os limites da firma nas suas fronteiras horizontais e verticais. O objetivo deste artigo é evidenciar fatores que influenciam na escolha da forma de investimento em crescimento (orgânico ou inorgânico) das indústrias brasileiras. Como metodologia adotou-se como consciência epistemológica o paradigma positivista quantitativo, e quanto aos objetivos, o método desenvolvido nesta pesquisa é

\footnotetext{
${ }^{1}$ Artigo recebido em 17.02.2013. Revisado por pares em 29.11.2013. Reformulado em 08.12.2013. Recomendado para publicação em 26.12.2013 por Carlos Eduardo Facin Lavarda. Publicado em 30.12.2013. Organização responsável pelo periódico: FURB.
} 
descritivo e explicativo. O problema de pesquisa a ser respondido nesta pesquisa foi: Existem fatores que podem ser considerados determinantes para escolher entre investimento em crescimento orgânico e investimento em crescimento inorgânico, como alternativas de expansão das empresas? A população da pesquisa é composta 93 indústrias brasileiras de capital aberto, analisadas no período de 1995 a 2008, com dados secundários, obtidos no "economática". Utilizaram-se dois modelos econométricos: 1) Painel Binomial de Dados, e 2) Logit Multinomial. As variáveis exógenas que apresentaram significância estatística positiva (5\%) foram: folga financeira e crescimento das vendas no Painel Binomial e no Logit Multinomial; grau de rentabilidade apresentou significância estatística negativa (5\%) no Logit Multinomial. Pode-se afirmar que folga financeira aumenta probabilidade de crescimento inorgânico. Maior nível de vendas igualmente aumenta a probabilidade de investimento em crescimento inorgânico. Quanto maior a rentabilidade, menores crescimento inorgânico.

Palavras-Chave: Indústria, Crescimento Orgânico, Crescimento Inorgânico.

\section{ABSTRACT}

Different theories about the firm's investment decision, whether or not neoclassical character, tend to consider the influence of organizational structures in determining investment. In modern corporations, to business strategies and organizational structures are taken into account the boundaries of the firm in its horizontal and vertical borders. The purpose of this article is to highlight factors that influence the choice of the form of investment in growth (organic or inorganic) of Brazilian industries. The methodology adopted as the epistemological awareness quantitative positivist paradigm, and about the objectives, the method developed in this research is descriptive and explanatory. The research question to be answered in this research was: There are factors that can be considered crucial to choose between investing in organic growth and inorganic growth in investment as alternative business expansion? The research population consists of 93 publicly traded Brazilian companies analyzed in the period from 1995 to 2008, with secondary data, obtained in "Economática." We used two econometric models: 1) Panel Data Binomial, and 2) Multinomial Logit. The exogenous variables that were statistically significant positive (5\%) were: financial slack and sales growth in Panel Binomial and Multinomial Logit; degree of profitability statistically significant negative (5\%) in the Multinomial Logit. It can be argued that financial slack increases probability of inorganic growth. Higher level sales also increases the likelihood of inorganic growth in investment. The higher profitability, lower inorganic growth.

Keywords: Industries. Organic growth. Inorganic growth.

\section{RESUMEN}

Diferentes teorías acerca de la decisión de inversión de la empresa, sea o no el carácter neoclásico, tienden a considerar la influencia de las estructuras organizativas en la determinación de la inversión. En las sociedades modernas, a las estrategias de negocio y estructuras de organización se consideran los límites de la empresa en sus bordes horizontales y verticales. El propósito de este artículo es destacar los factores que influyen en la elección de la forma de inversión en el crecimiento (orgánico o inorgánico) de las industrias brasileñas. La metodología fue adoptada como la conciencia cuantitativa paradigma positivista epistemológico, y sobre los objetivos, el método desarrollado en esta investigación es de tipo descriptivo y explicativo. El problema de investigación a ser respondidas en esta investigación fue: Hay factores que pueden ser considerados cruciales para elegir entre invertir en el crecimiento orgánico y el crecimiento inorgánico en la inversión como la expansión de negocios alternativa? La población de la investigación consiste en 93 industrias brasileñas que cotizan 
en bolsa analizadas en el periodo 1995-2008, con datos secundarios obtenidos en " Economática ". Se utiliza dos modelos econométricos: 1 ) Binomio de datos del panel, y 2) multinomial logit. Las variables exógenas que mostraron significación estadística positiva ( $5 \%$ ) fueron: la holgura financiera y crecimiento de las ventas en binomial y multinomial Panel Logit; grado de rentabilidad mostró negativa estadísticamente significativa ( $5 \%$ ) en el Logit multinomial. Se puede afirmar que la holgura financiera aumenta la probabilidad de crecimiento inorgánico. Mayor nivel de ventas también aumenta la probabilidad de que la inversión en el crecimiento inorgánico. La mayor rentabilidad, el crecimiento inorgánico inferior.

Palabras clave: Industria. Crecimiento orgánico. Crecimiento inorgánico.

\section{INTRODUÇÃO}

Nas Corporações modernas, para as estratégias empresariais e as estruturas organizacionais, são levados em consideração os limites da firma nas suas fronteiras horizontais e verticais, para criar ou ainda aumentar a quantidade de produtos para o mercado (CHANDLER, 1990; BESANKO et al, 2004; BARNEY, 1991).

Este artigo trata da forma de crescimento das indústrias brasileiras, especificamente aquele por meio de investimentos em aquisições de empresas, denominado de Crescimento Inorgânico, e o crescimento por meio de investimentos no núcleo das empresas, denominado de Crescimento Orgânico.

Hess (2007) define o crescimento orgânico (organic growth) como aquele alcançado quando a empresa assume compromisso com a satisfação do cliente, o engajamento de funcionários, núcleo da rentabilidade. Desta forma, o crescimento orgânico é uma estratégia de longo prazo para qualquer empresa. $O$ crescimento orgânico representa o dinamismo e a vitalidade do negócio, obtido por meio dos recursos internos da empresa ou financiados por meio de capital de terceiros.

Já o crescimento inorgânico (inorganic growth) é representado pela expansão de um negócio conquistado por meio de operações de concentração como fusões, aquisições, alianças, joint ventures o outros tipos de aliança. Conforme a especificidade de determinados setores, esse crescimento pode ocorrer devido a diretivas legais, impacto de alterações cambiais e conjuntura internacional. Em oposição ao crescimento orgânico, este tipo de expansão faz com que empresas atinjam de forma rápida dimensões que levariam mais tempo para atingir crescendo organicamente (HESS, 2007).

Sob esse ponto de vista, o crescimento orgânico representa um desafio, pois ao longo de grande parte da década de 1990, as empresas perceberam extraordinário crescimento em receitas e ganhos, e como esta tendência, gestores começaram a sentir pressão significativa para manter o crescimento orgânico contínuo (KAZANJIAN, HESS e DRAZIN 2006).

Segundo Kayo, Patrocínio e Martin (2008), as teorias econômica, financeira e estratégica sugerem muitos motivos para a escolha de aquisições como alternativas de crescimento inorgânico. Alguns desses motivos incluem poder de mercado, busca por sinergia operacional e financeira, aproveitamento de benefícios fiscais e substituição de gestores ineficientes (CAPRON e PISTRE, 2002). Os mesmos autores ainda citam o aumento do poder de Mercado, promoção de sinergia operacional e financeira, aproveitamento de ganhos com impostos e superação de gerenciamento com objetivos ineficientes.

Estudos relacionados ao crescimento orgânico se referem como posição estratégica e medição da performance financeira. Entre as questões de estudos foi levantado se o crescimento orgânico é prioritário; e se os recursos e práticas estão associadas à empresas bem-sucedidas (KAZANJIAN, HESS e DRAZIN, 2006). 
Estudo recente sobre crescimento orgânico analisa os determinantes do desempenho e elementos organizacionais (JOYCE, NOHRIA, e ROBERSON, 2003).

Partindo-se do pressuposto de expansão da firma, este artigo busca responder ao seguinte problema de pesquisa: Existem fatores que podem ser considerados determinantes para escolher entre investimento em crescimento orgânico e investimento em crescimento inorgânico como alternativa de expansão das indústrias brasileiras?

O objetivo deste artigo é evidenciar os fatores que podem influenciar na escolha da forma de investimento no crescimento das indústrias brasileiras, ou seja, investimento em crescimento orgânico ou investimento em crescimento inorgânico.

A justificativa para a escolha deste tema se baseia em Mulherin e Boone (2000) onde comparam modelos de indústria com efeitos na geração de riqueza por meio das aquisições e desinvestimentos. Seus resultados suportam sinergia sobre explicações teóricas de agência para aquisições e desinvestimentos, embora eles não testem diretamente os determinantes da escolha entre uma alternativa e outra (VILLALONGA e MCGAHAN, 2005).

Existem alguns estudos que analisam a escolha entre aquisições e Greenfield (BROUTHERS e BROUTHERS, 2000; HARZING, 2002; MEHMET, EKREM e KEITH, 2008); entre aquisições, Brownfield, e Greenfield (CHENG, 2006); entre aquisições, joint ventures e alianças contratuais como determinantes de escolha entre diferentes estratégias de crescimento (KAYO, KIMURA, PATROCINIO e OLIVEIRA NETO, 2009), entretanto não encontramos nenhum estudo empírico, nacional ou internacional, que fizesse essa abordagem de verificar a existência de fatores que pudessem ser considerados determinantes para escolher entre investimento em crescimento orgânico e investimento em crescimento inorgânico.

$\mathrm{O}$ artigo está estruturado da seguinte maneira: Introdução, onde apresenta, de forma geral, o assunto relacionado à pesquisa, seguido da construção do problema de pesquisa, objetivo e justificativa. Após é apresentada a Fundamentação Teórica, que trata das teorias que fundamentaram a pesquisa. As principais teorias abordadas são: Teoria da Agência, Teoria da Firma, Teoria do Custo de Transação e Teoria da Resource-Based View - RBV.

$\mathrm{Na}$ metodologia da Pesquisa explicitam os procedimentos para a pesquisa empírica em todas as fases, ou seja, desde a seleção da população/amostra até a análise dos dados com o modelo estatístico. Na sequência, são apresentados os resultados da pesquisa, com base na proposta feita na formulação do problema deste artigo com suas respectivas variáveis e testes.

E por fim, as considerações finais, que estão relacionadas à pesquisa empírica, além de sugestões para novas pesquisas.

\section{FUNDAMENTAÇÃO TEÓRICA}

\subsection{Rentabilidade}

Argumentos da teoria de agência explicam porque gerentes podem engajar em transações de boundary-spanning (controle de limites), mesmo quando essas transações incorrem grande risco e podem ser prejudiciais para o valor das ações (VILLALONGA e McGAHAN, 2005).

Leland e Pyle (1977) têm como ponto de partida as curvas de utilidade e riqueza para evidenciar que a decisão de investimentos por parte do empreendedor permite ao mercado exterior uma análise da situação interna dos projetos. Em outras palavras, o fato de o empreendedor ter conhecimento assimétrico permite que ele considere a porção que deseja reter de capital próprio e de terceiros em proporção tal que maximize sua utilidade própria.

A decisão de investimento é o processo de avaliar e selecionar investimentos em longo prazo, que sejam coerentes com o objetivo da empresa de maximizar a riqueza de seus proprietários, proporcionando maior rentabilidade. 
De acordo com Iyer e Miller (2008), empresas que apresentam desempenho abaixo das expectativas tendem a buscar mudanças estratégicas para se recuperarem. Entre várias alternativas, empresas nessa situação podem mudar sua carteira de investimentos. Nesse contexto, fusões e aquisições tornam-se mecanismos apropriados já que oferecem às empresas adquirentes uma forma rápida de recuperação. Iyer e Miller (2008) mostram evidências de que empresas com baixo desempenho apresentam maior probabilidade de realizar aquisições, mas não chegam a comparar aquisições com outras formas de crescimento (por exemplo, crescimento orgânico). Entretanto, é razoável supor que a rentabilidade possa afetar, também, a decisão de escolha entre crescer orgânica ou inorganicamente. Como as aquisições proporcionam uma forma mais rápida de mudança, supõe-se que empresas com baixo desempenho apresentam maior probabilidade de crescer inorganicamente. Assim, formulou-se a seguinte hipótese:

$\mathbf{H}_{1}$ : A rentabilidade é determinante para a escolha da forma de investimento em crescimento orgânico ou inorgânico nas indústrias brasileiras.

\subsection{Gerenciamento do Risco}

Geralmente o risco é visto como um potencial para perdas, por outro lado também pode representar o potencial de oportunidade de negócios, pela aquisição de outra empresa, ou pela adoção de estratégia de crescimento por meio de investimento no crescimento orgânico.

No processo decisório o risco é definido como sendo a variação na distribuição de resultados possíveis, suas probabilidades e seus valores subjetivos (MARCH e SAPHIRA, 1987). Matematicamente o risco pode ser expresso como sendo a probabilidade de ocorrência de perdas ou ganhos, multiplicada por sua respectiva magnitude (JAAFARI, 2001).

O gerenciamento do risco envolve a busca da identificação das ameaças e a análise da probabilidade destas ameaças realmente ocorrerem. A função da gestão de riscos é gerenciar a situação de "o que aconteceria se essas ameaças se materializassem" (BORODZICZ, 2005). Trata-se, também, da busca pela minimização da probabilidade de ocorrência da ameaça levando a efeitos indesejados, por meio do desenvolvimento, implementação e operação de controles internos que atenuam, fogem ou transferem os riscos.

O risco também pode ser visto como a possibilidade de falência. Nesse contexto, empresas ameaçadas por dificuldades financeiras evitam realizar operações de aquisição (Iyer e Miller, 2008). Aquisições de empresas, particularmente aquelas que visem alterar a carteira de investimentos da adquirente, podem ser mais arriscadas do que o crescimento orgânico já que aquelas podem alterar significativamente as atividades de uma empresa. Assim, supõe-se que o risco do negócio aumente a probabilidade de se realizar investimentos em crescimento orgânico. Assim formulou-se a seguinte hipótese:

$\mathbf{H}_{2}$ : O risco do negócio é determinante para a escolha da forma de investimento em crescimento orgânico ou inorgânico nas indústrias brasileiras.

\subsection{Folga Financeira}

Ao se realizar investimentos em crescimento orgânico ou inorgânico é imprescindível analisar a folga financeira da investidora, independentemente se ela utilizará capital próprio ou de terceiros. De acordo com Iyer e Miller (2008), empresas com folga financeira apresentam a tendência a experimentar e assumir maior risco. Nesse contexto, os investimentos em aquisições (supostamente mais arriscados que o orgânico) seriam privilegiados. Assim, supõese que quanto maior a folga financeira, maior a probabilidade de crescimento inorgânico. Sob esse ponto de vista a hipótese formulada é:

$\mathbf{H}_{3}$ : A folga financeira é determinante para a escolha da forma de investimento em crescimento orgânico ou inorgânico nas indústrias brasileiras. 


\subsection{Risco de Falência}

Os custos de falência diretos dizem respeito ao processo legal que envolve a reorganização de uma empresa falida, enquanto os custos de falência indiretos não estão relacionados de forma direta com a reorganização da empresa, podendo surgir entre empresas com problemas financeiros, ou entre aquelas empresas que estão próximas da falência, mas que talvez não cheguem a falir (GRINBLATT e TITMAN, 2005).

Comumente a falência dificulta o relacionamento com clientes e fornecedores, o que pode acarretar perda de receita, aumento de exigências por parte dos fornecedores e dificuldades de obter novos recursos para financiar projetos.

O risco de falência exerce um efeito negativo sobre o valor da empresa. No entanto, não é o risco de falência em si que reduz o valor. Na verdade, são os custos associados à falência que o reduz.

Conforme Copeland e Weston (1992) os custos de falência podem alterar o valor de mercado de uma empresa porque em um processo de falência, uma terceira parte, que não os acionistas e os credores, receberia também parte dos desembolsos, fracionando o fluxo de caixa, o que evidentemente diminuiria o seu valor presente. Sob essa ótica a hipótese formulada é:

$\mathbf{H}_{4}$ : O risco de falência é determinante para a escolha da forma de investimento em crescimento orgânico ou inorgânico nas indústrias brasileiras.

\subsection{Endividamento}

As organizações têm atribuído aos administradores financeiros o objetivo de maximizar a riqueza dos proprietários, por meio da função financeira, a qual tem sob sua incumbência os três principais tipos de decisão: decisões de investimento, decisões de financiamento e as decisões de distribuição de dividendos (BREALEY e MYERS, 2005).

A teoria do pecking order, por exemplo, baseia-se na idéia de que os administradores se pautam por uma hierarquia para definir que recursos serão utilizados para financiar seus projetos. Mesmo que os administradores sigam tal hierarquia, não fica invalidada a idéia de um nível ótimo de endividamento na linha proposta pela denominada teoria do Trade - Off.

A escolha do Trade-off abrange dois fatores que atuam como forças contrárias: por um lado, há o efeito das economias fiscais, agindo no sentido de incentivar o uso de dívidas; por outro lado, o efeito dos custos de falência esperados, que surgem da maior propensão de uma empresa tornar-se inadimplente diante de uma situação de alto endividamento relacionado à investimentos, seja em aquisições ou em crescimento orgânico.

Para verificar quais seriam os principais fatores determinantes do endividamento das empresas brasileiras Famá e Perobelli (2001) replicaram o modelo apresentado por Titman e Wessels (1988); usando análise fatorial evidenciaram relação negativa entre o grau de endividamento no curto prazo e as variáveis "tamanho e crescimento dos ativos". Os resultados evidenciaram que empresas de menor porte tendem a se financiar mais com recursos de curto prazo, enquanto empresas de maior porte são menos dependentes de dívidas de curto prazo. Sob essa perspectiva formulou-se a seguinte hipótese:

H5: $_{5}$ O nível de endividamento é determinante para a escolha da forma de investimento em crescimento orgânico ou inorgânico nas indústrias brasileiras.

\subsection{Nível das Vendas}

Durante a expansão do modelo neoclássico sobre a teoria da firma, uma abordagem diferenciada foi desenvolvida e denominada de economia do custo de transação, iniciada por Coase (1937). Essa abordagem investiga a existência das firmas a partir dos custos de refletir, planejar e contratar que acompanhava qualquer transação (HART, 1996).

Economias de tamanho se mostram quando uma firma de maior porte, em relação ao seu tamanho, pode não somente produzir e vender bens e serviços de forma mais eficiente do 
que firmas menores, mas também introduzir mais eficientemente maiores quantidades ou produtos novos (PENROSE, 1959).

A hipótese de que firmas têm um contínuo estímulo para expandir-se, não levando em consideração seus tamanhos absolutos contrasta com a noção de um tamanho ótimo para elas. O tamanho da firma era determinado pelos custos de transação e pela capacidade gerencial, na medida em que quanto maior a capacidade gerencial, maior o número de transações que poderiam incorporar-se à firma (COASE, 1937).

Uma vez que a expansão tenha se completado, a justificativa original pode ter desaparecido por completo na medida em que novas oportunidades de crescimento vão aparecendo e sendo aproveitadas. Sob esta perspectiva, formulou-se a seguinte hipótese:

$\mathbf{H}_{6}$ : O nível das vendas é determinante para a escolha da forma de investimento em crescimento orgânico ou inorgânico nas indústrias brasileiras.

\subsection{Tamanho da Empresa}

Segundo Solow (1985), o objetivo da economia dos custos de transação é entender a transação como unidade de análise e a firma como uma governance structure (estrutura de gestão), onde o que importa é a estrutura interna, os incentivos e os mecanismos de controle mais do que a função de produção.

A teoria dos custos de transação aborda o problema da organização, neste estudo com o constructo de forma de crescimento, como sendo um problema contratual, onde a firma é vista como um complexo de contratos, que podem ser explícitos ou implícitos, e onde ambos estão sujeitos aos custos de transação, que podem ser classificados como ex-ante e ex-post (WILLIANSON, 1985).

Coase (1972) discute que custos internos de organização são propensos a serem mais altos quando existe dissimilaridade entre as atividades da transação e da firma, porque a falta de um precedente dentro da firma cria maiores demandas na estrutura da organização. Por outro lado, quanto maior o nível de diversificação prévia, maior a probabilidade de existência de atributos comuns com as atividades de transação, e assim maior a probabilidade de integrar as atividades após o fato (VILLALONGA e McGAHAN 2005).

A teoria de custo de transação estipula que uma firma escolhe adquirir ativos quando os custos de progresso na condução dos negócios no mercado estão mais altos que os custos das atividades organizacionais dentro da firma (COASE, 1937; WILLIAMSON, 1975, 1985).

Hennart e Park (1993) argumentam que várias firmas ou corporações preferem aquisições porque elas possuem um sofisticado sistema de controle de governo que pode ser explorado por meio de aquisições estrangeiras, desta maneira fornecendo uma eficiência organizacional. Com base nesses argumentos, foi construída a seguinte hipótese:

$\mathbf{H}_{7}$ : O tamanho da firma é determinante para a escolha da forma de investimento em crescimento orgânico ou inorgânico nas indústrias brasileiras.

\subsection{Recursos Tangíveis e Intangíveis na Perspectiva da RBV}

Wilk (2000), fazendo uma releitura do conceito de recurso proposto por Grant, apresenta outra classificação, considerando a existências de recursos tangíveis, que podem ser observados e avaliados de forma clara e dos recursos intangíveis, que não são assim observados e quantificados.

Os recursos intangíveis são pontuados pela Resource-Based View como os principais estimuladores da sustentação das diferenças entre os desempenhos das empresas. Diferentes estudos relacionados à literatura sobre RBV têm empregado termos diferentes para se referirem a estes recursos, tais como capacidades, competências essenciais ou conhecimento (VILLALONGA, 2004). 
Para Balakrishnan e Fox (1993), os investimentos em ativos intangíveis contribuem para variações na alavancagem da firma. Afirmam ainda que se tal relacionamento fosse encontrado, indicaria que a estratégia, que pode ter efeito no nível de recursos intangíveis em uma firma, pode ajudar determinar a sua estrutura de capital em relação a tamanho. Diante desses argumentos, testa-se a seguinte hipótese.

$\mathbf{H}_{8}$ : A intangibilidade dos ativos da investidora é determinante para a escolha da forma de investimento em crescimento orgânico ou inorgânico nas indústrias brasileiras.

\subsection{Estudos Similares ou correlatos}

Um estudo para identificar oportunidades de crescimento da firma foi proposto originalmente por Ansoff (1965), conhecida como matriz de crescimento produto/mercado, considerando: (a) penetração, por meio de ganho de market share dos produtos vigentes sob mercados de atuação; (b) desenvolvimento de novos produtos; (c) desenvolvimento de novos mercados, e (d) diversificação de negócios. Esta diversificação de negócios pode ainda ser caracterizada como relacionada ou não aos negócios atuais. (GARCEZ, 2006). Mesmo analisando as oportunidades de crescimento da firma, não foram analisados os fatores que levam a escolher a forma de crescimento, ou seja, com investimento em crescimento orgânico ou em crescimento inorgânico.

Estudos empíricos evidenciam que as aquisições proporcionam a oportunidade para a transferência de recursos, capacidade e experiência pessoal com críticas entre organizações (AHUJA e KATILA, 2001; KARIM e MITCHELL, 2000). Apesar das operações de aquisições poderem criar tensões na integração, também podem enriquecer o conhecimento base e quebrar a rigidez da aquisição de empresas (VERMEULEN e BARKEMA, 2001).

Para Hitt, Ireland e Hoskisson (2005), uma razão importante para as aquisições é obter maior poder de mercado, que existe quando uma firma é capaz de vender seus bens e serviços acima dos níveis competitivos ou ainda, quando os custos de suas atividades principais ou de apoio são inferiores aos dos competidores. O poder de mercado geralmente é derivado do tamanho da firma e de seus recursos e capacidades para competir no mercado.

As operações de aquisições de empresas são excessivamente complexas, pois envolvem vários intervenientes nas negociações ao mesmo tempo. Nesse cenário, é fácil observar que o processo comporta riscos elevados para a empresa adquirente, como por exemplo, o de pagar um preço muito alto ou, mesmo depois de fechado o negócio, encontrar sérios obstáculos na fusão das empresas, obrigando as adquirentes a definir uma estratégia para que o risco da operação não aumente.

Estudos têm tentado relacionar a aquisição com atividade empresarial medida por ciclo de produção industrial, preços das ações, incorporações empresariais e taxas de juros (WESTON, SIU e JOHNSON, 2001). As conclusões desses estudos são de que a atividade de aquisição é cíclica e praticamente coincidente com o movimento do preço das ações; já em relação ao crescimento interno o problema que as firmas enfrentam reside em tentar determinar e buscar metas de crescimento.

Embora pesquisadores tenham dado considerável atenção para as motivações econômicas que levam às aquisições, a escolha do que leva a tomar as decisões sobre fazer aquisição (Timing of acquisitions) ainda é relativamente pouco estudada. Várias pesquisas nacionais e internacionais têm tratado de Fusões e Aquisições com vista à criação de valor e do crescimento da firma sob a perspectiva de análise de desempenho e criação de valor.

Em sua pesquisa Kazanjian, Hess e Drazin, (2006) concentram-se em questões relacionadas com a estratégia para o crescimento orgânico, ressaltando questões refletidas nas experiências da Siemens, UPS, The Home Depot, e SYSCO. Essas questões incluem: (1) se o crescimento orgânico é definido como a estratégica prioritária das empresas (2) qual é a relação 
de aquisições para o crescimento orgânico e (3) se o alinhamento de recursos e práticas está associado às empresas de crescimento bem-sucedido.

Estudo recente sobre os determinantes do desempenho, com uma amostra de 200 empresas industriais busca investigar elementos organizacionais que influenciam no crescimento orgânico (JOYCE, NOHRIA, e ROBERSON, 2003). O estudo original foi desenhado para identificar quatro empresas por setor, fazendo o acompanhamento do seu desempenho ao longo de um período de dez anos. O crescimento é fortemente enfatizado na estratégia da empresa em todo o período do estudo. Um foco estratégico no crescimento orgânico supõe uma definição clara e específica, mas a medição do crescimento orgânico é uma tarefa complexa (JOYCE, NOHRIA, e ROBERSON, 2003).

Embasado na análise dessas pesquisas empíricas, não foi encontrado nenhuma evidência de estudos que analisasse os fatores determinantes para escolher a estratégia de investimento em crescimento orgânico e em inorgânico.

\section{METODOLOGIA DA PESQUISA}

Para responder o problema de pesquisa proposto neste artigo utilizou-se como método de pesquisa: Quanto aos objetivos, o método desenvolvido nesta pesquisa foi descritivo e explicativo; Quanto aos procedimentos, a pesquisa foi documental; e Quanto a abordagem do problema, a pesquisa foi quantitativa.

No estudo descritivo se sabe que as características ou fenômenos existem e se quer descrever características de determinada população ou fenômeno, ou o estabelecimento de relação entre variáveis (SEKARAN, 1992). Neste artigo busca-se descrever com relativa profundidade os objetos em estudo, neste caso, as empresas do setor industrial do Brasil que tem suas ações negociadas na Bolsa, buscando prováveis evidências relacionadas com a escolha das formas de investimento em crescimento, ou seja, orgânico ou inorgânico.

O método explicativo tem por finalidade identificar fatores que determinam ou contribuem para a ocorrência dos fenômenos. A pesquisa explicativa além de registrar, analisar, classificar e interpretar os fenômenos estudados, ainda procura identificar seus fatores determinantes (GIL, 1999; ANDRADE, 2002).

Para satisfazer ao caráter explicativo desta pesquisa, busca-se embasamento no modelo teórico de temas significativos para o estudo. Consideram-se como modelos fundamentais para esta pesquisa, o estudo relacionado com o crescimento orgânico e o crescimento inorgânico.

\subsection{Amostra do Estudo}

A amostra deste estudo é composta pelas industrias brasileiras de capital aberto que possuem ações listadas na Bolsa de Valores de São Paulo (BOVESPA), com investimento em pelo menos em uma da formas de crescimento (orgânico ou inorgânico), cujas informações econômico-financeiras estejam contidas na fonte de dados secundária "Economática" no período de 1995 a 2008. Sendo o foco do trabalho nas indústrias, excluiu-se da amostra todas as empresas comerciais, instituições financeiras e empresas de serviços listadas na Bolsa.

O banco de dados das indústrias brasileiras listadas na BOVESPA é constituído de 151 empresas; entretanto, como o objetivo deste trabalho foi estudar os fatores que influenciam na estratégia de escolha da forma de crescimento dessas empresas, entre crescimento orgânico ou crescimento inorgânico, foram selecionadas para este estudo somente as indústrias que tiveram anúncio de pelo menos uma das formas de investimento em crescimento, totalizando 93 indústrias.

Destas, coletaram-se dados referentes ao período de 1995 a 2008 para calcular as 8 variáveis independentes, totalizando 830 observações. 
Os anúncios dos planos de investimentos das indústrias foram extraídos do Economática no período de $1 .^{\circ}$ de janeiro de 1995 a 31 de dezembro de 2008, na base de dados "Fatos Relevantes".

Os Fatos Relevantes são normatizados pela Instrução CVM no 358, de 3 de janeiro de 2002, e alterados pela Instrução CVM n 449 , de 15 de março de 2007. Depois de selecionadas as indústrias brasileiras listadas na BOVESPA, foram pesquisados todos os anúncios com propósito específico de verificar se atendiam a intenção desta pesquisa.

\subsection{Variável Dependente}

A operacionalização desta variável aconteceu em duas análises distintas: Em um primeirto momento como Binomial assumindo como crescimento orgânico o vetor (0) e o crescimento inorgânico o vetor (1) no Painel Binomial de Dados. Na segunda análise como Multinomial assumindo os vetores (1) para crescimento orgânico; (2) para crescimento inorgânico; (3) para orgânico e inorgânico; e (4) não para para orgânico e inorgânico.

Assim na base de dados para definição da variável dependente atribuiram-se os seguintes vetores:

Tabela 1 - Composição da Variável Dependente para o Painel Binário de Dados

\begin{tabular}{lccc}
\hline $\begin{array}{l}\text { Definição VD } \\
\text { Regressando }\end{array}$ & símbolo & & Def. \\
\hline Para o Crescimento Orgânico & CO & & $\mathbf{0}$ \\
Para o Crescimento Inorgânico & CI & $\mathbf{1}$ \\
\hline
\end{tabular}

Fonte: Elaborada pelos autores

Tabela 2 - Composição da Variável Dependente para o Logist Multinomial

\begin{tabular}{lccc}
\hline $\begin{array}{l}\text { Definição VD } \\
\text { Regressando }\end{array}$ & símbolo & & Def. \\
\hline Para o Crescimento Orgânico & CO & & $\mathbf{1}$ \\
Para o Crescimento Inorgânico & CI & & $\mathbf{2}$ \\
Para Cres. Orgânico e Inorgânico & CO e CI & & $\mathbf{3}$ \\
E para Ausência de CO e CI & ñCO e ñCI & $\mathbf{4}$ \\
\hline
\end{tabular}

Fonte: Elaborada pelos autores

\subsection{Visão Resumida das Variáveis Independentes}

Para que se possa construir a matriz de dados, são operacionalizadas para cada uma das empresas e período analisado, 8 variáveis independentes métricas, conforme podem ser observadas de forma resumida na Tabela 3.

Por se tratar de um estudo, onde não existem modelos correlatos ou similares, esta pesquisa faz uma tentativa e também uma provocação para novas propostas de estudo, assim buscou-se com base na fundamentação teórica, possíveis variáveis para serem usadas e testadas como fatores que podem ser determinantes para a escolha entre as formas de investimentos propostos.

Com a escolha destas variáveis, como fatores determinantes para escolha da forma de investimento, não podemos afirmar de forma categórica que são as mais apropriadas, porém com essa proposição, espera-se que outros estudos também testem outras variáveis, contribuindo também para o avanço da ciência. 
Tabela 3 - Determinantes da escolha da forma de crescimento

\begin{tabular}{llll}
\hline \multicolumn{1}{c}{ Variável } & Resumo & Tipo & \multicolumn{1}{c}{ Mensuração } \\
\hline 1. Grau de Rentabilidade & Gr_Rent & métrica & $\begin{array}{l}\text { Calculado por meio do índice de Rentabilidade } \\
\text { no Balanço Patrimonial das empresas }\end{array}$ \\
\hline 2. Risco do Negócio & Ris_Neg & métrica & $\begin{array}{l}\text { Índicie do EBIT/Ativo Total diminuido da média } \\
\text { do período analizado }\end{array}$ \\
\hline 3. Folga Financeira & Fol_Fin & métrica & $\begin{array}{l}\text { Indice de Liquidez Corrente no Balanço } \\
\text { Patrimonial das Empresas. }\end{array}$ \\
\hline 4. Risco de Falência & Ris_Fal & métrica & $\begin{array}{l}\text { Aplicação do modelo conforme equação abaixo } \\
\text { Industrias Brasileiras }\end{array}$ \\
\hline 5. Nível de Endividamento & Niv_End & métrica & $\begin{array}{l}\text { Calculado por meio do índice de Endividamento } \\
\text { Ativo curto e longo/ Passivo curto e longo prazo }\end{array}$ \\
\hline 6. Tamanho das Vendas & Log_Ven & métrica & $\begin{array}{l}\text { Cálculo do Logarítimo das vendas disponível } \\
\text { na Dem. do Res. Do Exerc. DRE (Economática) }\end{array}$ \\
\hline 7. Tamanho do Ativo & Log_At & métrica & $\begin{array}{l}\text { Cálculo do Logarítimo do Ativo disponível } \\
\text { no Balanço Patrimonial das empresas }\end{array}$ \\
\hline 8. Intangibilidade do Ativo & Tobin'sQ & métrica & $\begin{array}{l}\text { Calculado dividindo-se o valor de mercado da } \\
\text { empresa pelo valor contábil da empresa }\end{array}$ \\
\hline
\end{tabular}

Fonte: Elaborada pelos autores

\subsection{Modelos}

Entretanto, para o período compreendido, nem todas as indústias possuem os dados econômico-financeiros para todos os anos do período analisado, o que implica na utilização de um painel binário não balanceado (ou painel desiquilibrado), onde o número de observações não é igual para todas as indústrias.

$\mathrm{Na}$ literatura de econometria existem duas grandes classes de modelos de análise de dados em painel: os modelos estáticos, e os modelos dinâmicos.

Resumidamente os dados em painel modelam ou explicam porque os indivíduos ou companhias se comportam de modos diferentes, assim como modelam ou explicam porque os indivíduos ou as companhias se comportam de modo diferente em diferentes períodos de tempo (VERBEEK, 2001).

Para permitir a análise dos dados no presente estudo utilizou-se a modelagem econométrica por meio de Painel Binário de Dados e Logit Multinomial, representada pelas seguintes equações:

\section{Modelo do Painel Binário:}

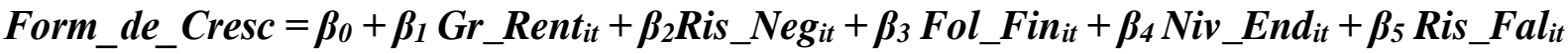
$+\beta_{6} \log \bar{V}_{-} \mathrm{n}_{i t}+\beta_{7} \log \_A t_{i t}+\beta_{8}$ Tobin's_Q $Q_{i t}+\mu_{i t}$

Form_de_Cresc $=$ Variável Endógena Binária que recebe (0) para crescimento orgânico e (1) para crescimento inorgânico .

$\mathrm{Na}$ equação acima o $\beta_{0}$ representa o intercepto, os demais $\beta_{k}$ representam os coeficientes de cada variável regressora, $i$ representa a i-ésima unidade de corte transversal, $t$ representa o tésimo período de tempo, $\mu_{i t}$ representa o termo de erro aleatório.

Modelo Logit Multinomial:

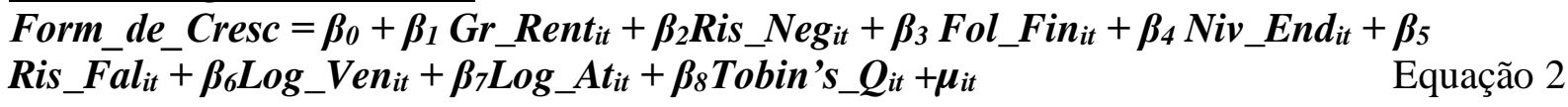


Em ambos os modelos, as variáveis foram as seguintes:

Grau de Rentabilidade $=G r_{-}$Rent $=\frac{E B I T}{V e n d a}$

Risco do Negócio $=$ Ris_Neg $=\lambda$ com $\frac{E B I T}{A T}=\mu$ e $\lambda=\frac{\sum\left(P_{1}+P_{2}+\ldots P_{n}\right)}{P_{n}}-\mu$

(Conforme CORREA, BASSO e NAKAMURA 2007).

Folga Financeira $=$ Fol_Fin $=\frac{A C}{P C}$

Nível de Endividamento $=$ Niv_End $=\frac{(\boldsymbol{A} \in \boldsymbol{A} \tilde{N})}{(\boldsymbol{P} \in \boldsymbol{P} \tilde{N})}$

Risco da Falência $=$ Ris_Fal $=0,722-5,124 E_{23}+11,016 L_{19}-0,342 L_{21}-0,048 L_{26}+$ $8,605 R_{13}-0,004 R_{29}$

Logaritmo das Vendas $=$ Log_Ven de base natural

Logaritmo do Ativo = Log_Ati de base natural

Intangibilidade do Ativo $=Q$ de Tobin $=\frac{V M E}{V C E}$

Na equação acima o $\beta_{0}$ representa o intercepto, os demais $\beta_{k}$ representam os coeficientes de cada variável regressora, $i$ representa a i-ésima unidade de corte transversal, $t$ representa o tésimo período de tempo, $\mu_{i t}$ representa o termo de erro aleatório. Neste artigo o modelo de painel utilizado foi o estático.

No caso da decisão quanto aos efeitos fixo ou aleatório, utilizou-se o teste conforme Hausman (1978). A hipótese nula $\left(\mathrm{H}_{0}\right)$ para o teste de Hausman configura que o erro específico do grupo não é correlacionado entre si e, portanto, o modelo de efeito aleatório é preferível. Um p-valor menor que o nível de significância de 5\% rejeita o efeito aleatório em favor do efeito fixo.

Quanto à caracterização do intercepto para o efeito fixo, utilizou-se o teste de BreuschPagan. O efeito fixo do modelo permite que a intercepção da regressão possa variar entre as unidades de corte transversal. $O$ teste $F$ é atribuído para a hipótese nula de que as interceptações não diferem entre si. Para tanto, a estatística de Lagrange Multiplier (LM) do teste BreuschPagan testa a hipótese nula de que o estimador Pooled OLS é adequado contra os efeitos aleatórios alternativos.

Tanto o teste de Hausman quanto o teste de Breush-Pagan podem ser esquematizados conforme apresentado por Richieri (2007). A Figura 1 apresenta de forma detalhada as hipóteses a serem testadas para se decidir sobre qual modelo utilizar: Modelo de Efeito Fixo ou Modelo de Efeito Aleatório.

As opções possíveis podem ser analisadas por meio da Figura 1. Evidencia-se os testes $F$ (Fischer), Breuch-Pagan (BP) e Hausman (H) para aceitar ou rejeitar as hipóteses nulas para verificar se os estimadores em painel estático são consistentes pelo Minimo Quadrado Ordinário - MQO (Ordinary Least Squares - OLS) ou pelo Minimo quadrado Generalizado MQG (Generalized Least Squares - GLS), conforme as hipóteses configuradas na fundamentação teórica. 
Figura 1 - Processo de escolha de estimadores em painéis de dados estáticos

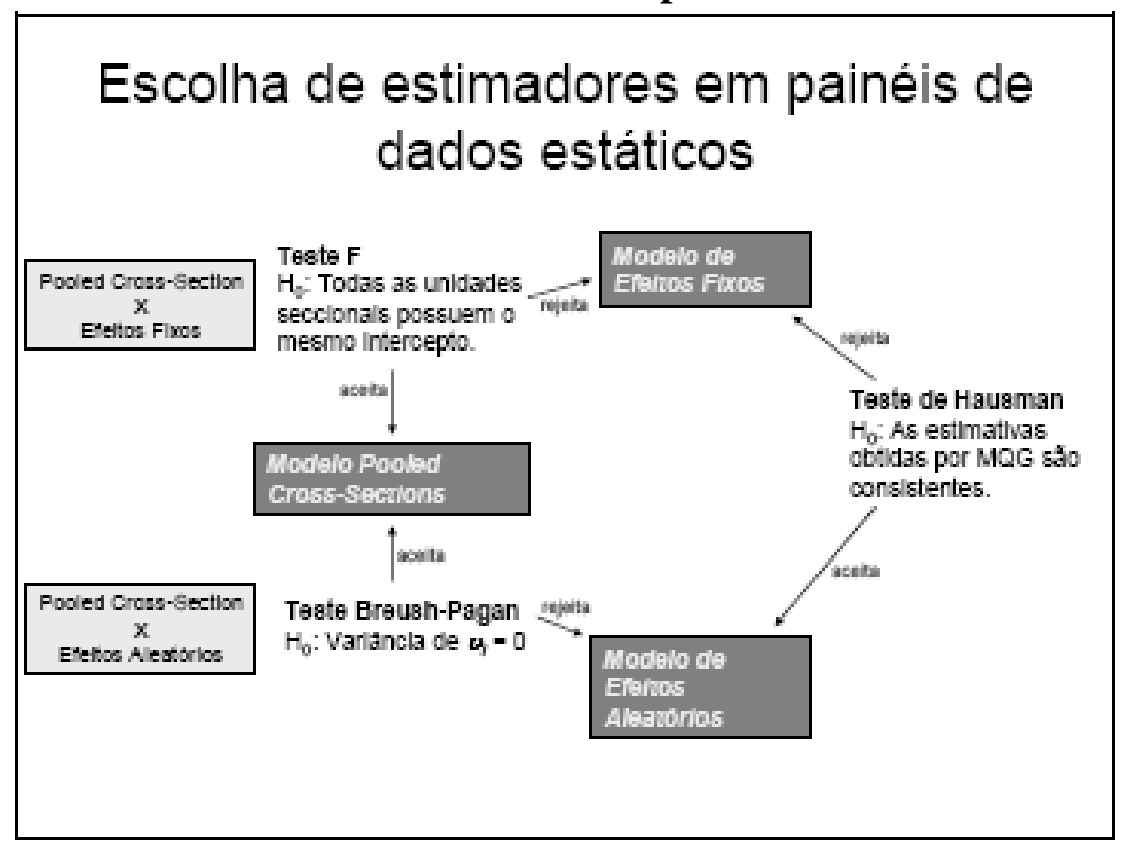

Fonte: Richieri (2007, p.118)

\section{RESULTADO DA PESQUISA}

\subsection{Resultados das Regressões}

Para facilitar a exposição da sequência com que foram realizadas as regressões para a variável dependente Forma_de_Cresc, todos os resultados estão consolidados de acordo com os modelos de regressão selecionados para este estudo.

Comparou-se os três modelos econométricos de regressão para o tipo estático em painel não balanceado: Efeito Fixo com erros padrão robusto, os Efeitos Aleatórios com Mínimos Quadrados Generalizados - MQG (Generalized Least Squares - GLS), e o Estimador de Equação Generalizada - Pooled (Generalized Equations Estimator - GEE). Realizou-se também a análise do Logit Multinomial. No quadro 1 é apresentada uma síntese dos resultados das regressões no modelo de painel estático.

\section{Quadro 1 - Valores $p$ das variáveis no painel binário não balanceado}

\begin{tabular}{|c|c|c|c|c|c|c|}
\hline \multirow[b]{4}{*}{ Variáveis } & \multicolumn{6}{|c|}{$\begin{array}{l}\text { Forma_de_Cresc em Painel Não Balanceado com } 93 \text { indústrias } \\
\text { Modelos adotados para as Regressões de Dados em Painel Binário }\end{array}$} \\
\hline & \multicolumn{2}{|c|}{1} & \multicolumn{2}{|c|}{2} & \multicolumn{2}{|c|}{3} \\
\hline & \multicolumn{2}{|c|}{$\begin{array}{c}\text { EF } \\
\text { Within }\end{array}$} & \multicolumn{2}{|c|}{$\begin{array}{c}\text { EA } \\
\text { MQG }\end{array}$} & \multicolumn{2}{|c|}{$\begin{array}{c}\text { GEE } \\
\text { Pooled }\end{array}$} \\
\hline & Coeficiente & p-value & Coeficiente & p-value & Coeficiente & p-value \\
\hline Const & -98529 & $<0,000 * * *$ & $-13,1773$ & $<0,000 * * *$ & $-9,8529$ & $<0,000 * * *$ \\
\hline Gr_Rent & 2,2628 & 0,492 & $-0,0325$ & 0,851 & $-0,2355$ & 0,264 \\
\hline Ris_Neg & $-3,1083$ & 0,561 & 0,7333 & 0,719 & 0,6237 & 0,464 \\
\hline Fol_Fin & 0,3599 & $0,113^{*}$ & 0,3582 & $0,012 * * *$ & 0,3346 & $0,000 * * *$ \\
\hline Ris_Fal & 0,1504 & 0,239 & $-0,2585$ & 0,714 & $-0,0359$ & 0,230 \\
\hline Niv_End & $-0,3204$ & 0,501 & $-0,2835$ & 0,370 & $-0,2835$ & 0,350 \\
\hline Log_Ven & 3,8923 & $0,016^{* * *}$ & 0,9508 & 0,207 & 0,7097 & 0,351 \\
\hline Log_At & 1,5826 & 0,244 & 0,7741 & 0,311 & 0,5298 & 0,418 \\
\hline Q_Tobin & 0,0018 & 0,752 & 0,0010 & 0,795 & 0,0007 & 0,871 \\
\hline
\end{tabular}

Fonte: Elaborado pelos autores 
Em um segundo momento analisou-se o Modelo Logit Multinomial usado quando a variável dependente em questão é nominal (um conjunto de categorias que não podem ser ordenados de forma significativa) e que consiste de quatro categorias.

\subsection{Análise do Painel Binário de Dados}

No modelo de efeito fixo, a variável Log_Ven (logarítimo das vendas) apresentou significância positiva (p-valor 0,0160). A variável Fol_Fin (folga financeira) apresentou significância positiva ao nível próximo de $10 \%$. Portanto, quanto maiores os valores destas variáveis, maior a probabilidade de ocorrência do investimento em crescimento inorgânico.

No modelo Pooled, somente a variável Fol_Fin (folga financeira) apresentou significância positiva (p-valor de 0,012 ) Assim quanto maior o seu valor, maior a probabilidade de ocorrência de investimento em crescimento inorgânico.

No modelo de efeito aleatório também a variável Fol_Fin (folga financeira) apresentou significância positiva (p-valor de 0,00 ). Logo, quanto maior o seu valor, maior a probabilidade de ocorrência de investimento em crescimento inorgânico.

Após os testes de Breusch-Pagan e Hausmam, o modelo que se apresentou como sendo o mais adequado pelo teste das hipóteses foi o modelo de Efeitos Aleatórios - EA, sendo comprovadamente o mais adequado para o estudo com painel binário não balanceado para as indústrias brasileiras de capital aberto, componentes da amostra.

As variáveis que não foram significantes para explicar a estratégia de investimento por meio da forma de crescimento foram: Gr_Rent (grau de rentabilidade), Ris_Neg (risco do negócio), Ris_Fal (risco de falência), Niv_End (nível de endividamento), Log_Ven (Logarítimo das vendas) no modelo de efeito aleatório, Log_At (logarítimo dos ativos) e Q_Tobin (Q de Tobin). O quadro 2 apresenta os resultados das regressões do Efeito Aleatório.

\section{Quadro 2 - Modelo 2: Efeito Aleatório usando 830 observações}

\begin{tabular}{|c|c|c|c|c|c|c|}
\hline Variáveis & Coeficiente & Std Err & $\boldsymbol{z}$ & $\boldsymbol{P}>\boldsymbol{z}$ & 95\% Conf & Interval \\
\hline Const & $-13,1773$ & 2,945 & $-4,47$ & $<0,000 * * *$ & $-18,9494$ & -74051 \\
\hline Gr_Rent & $-0,0325$ & 0,1729 & $-0,19$ & 0,851 & $-0,3716$ & 0,3064 \\
\hline Ris_Neg & 0,7333 & 2,0397 & 0,36 & 0,719 & $-3,2645$ & 4,7311 \\
\hline Fol_Fin & 0,3582 & 0,1426 & 2,51 & $0,012 * * *$ & 0,0786 & 0,6379 \\
\hline Ris_Fal & $-0,2585$ & 0,0704 & $-0,37$ & 0,714 & $-0,1639$ & 0,1122 \\
\hline Niv_End & $-0,2835$ & 0,3164 & $-0,90$ & 0,370 & $-0,9037$ & 0,3367 \\
\hline Log_Ven & 0,9508 & 0,7537 & 1,26 & 0,207 & $-0,5265$ & 2,4282 \\
\hline Log_At & 0,7741 & 0,7648 & 1,01 & 0,311 & $-0,7248$ & 2,2731 \\
\hline Q_Tobin & 0,0010 & 0,0041 & 0,26 & 0,795 & $-0,0070$ & 0,0092 \\
\hline
\end{tabular}

Fonte: Elaborado pelos autores

Quanto às hipóteses da pesquisa apresentam-se os seguintes resultados para o painel binário: A hipótese $\mathrm{H}_{1}$ foi rejeitada $\mathrm{A}$ rejeição desta hipótese leva a aceitação da hipótese alternativa onde a rentabilidade não é determinante para a escolha da forma de investimento em crescimento nas indústrias brasileiras.

A hipótese $\mathrm{H}_{2}$ foi rejeitada. A rejeição desta hipótese leva a aceitação da hipótese alternativa onde o risco do negócio não foi considerado como um dos fatores determinantes para se escolher a forma de investimento em crescimento orgânico ou inorgânico nas indústrias.

A hipótese $\mathrm{H}_{3}$ não foi rejeitada, ao nível de significância de 5\%. Verificou-se também que o sinal estimado é positivo, evidenciando que ao aceitar esta hipótese, há uma evidencia da relação entre a folga financeira com a forma de investimento em crescimento inorgânico. 
A hipótese $\mathrm{H}_{4}$ foi rejeitada. A rejeição desta hipótese leva a aceitação da hipótese alternativa onde o risco de falência não é significante para a escolha da forma de investimento em crescimento orgânico ou inorgânico nas indústrias brasileiras ao nível de $5 \%$.

A hipótese $\mathrm{H}_{5}$ foi rejeitada. A rejeição desta hipótese leva a aceitação da hipótese alternativa onde nível de endividamento não foi considerado como um dos fatores determinantes para se escolher a forma de investimento em crescimento orgânico ou inorgânico nas indústrias.

A hipótese $\mathrm{H}_{6}$ foi rejeitada. A rejeição desta hipótese leva a aceitação da hipótese alternativa onde o tamanho das vendas não é um fator determinante para escolher a forma de investimento em crescimento nas indústrias brasileiras pelo painel binário.

A hipótese $\mathrm{H}_{7}$ foi rejeitada. A rejeição desta hipótese leva a aceitação da hipótese alternativa onde o tamanho dos ativos da investidora não é determinante para a escolha da forma de investimento em crescimento nas indústrias brasileiras.

A hipótese $\mathrm{H}_{8}$ foi rejeitada. A rejeição desta hipótese leva a aceitação da hipótese alternativa onde a intangibilidade dos ativos, medida pelo $\mathrm{Q}$ de Tobin não é determinante para a escolha da forma de investimento em crescimento nas indústrias brasileiras.

\subsection{Análise do Logit Multinomial}

Neste estudo, a definição da variável dependente para análise pela modelagem Logit Multinomial se deu por meio de vetores definidos como regressores a partir de quatro formas de crescimento: 1 para crescimento orgânico, 2 para crescimento inorgânico, 3 para crescimento orgânico e inorgânico, e 4 para nenhuma forma de crescimento. Partindo-se desse pressuposto, foi necessária a utilização do Logit Multinomial para fazer inferência em cada forma de crescimento para verificar as características de cada uma, em relação à semelhança entre os vetores.

O quadro 3 evidencia o resultado encontrado conforme o modelo do Logit Multinomial, com erro padrão robusto. Estatisticamente em cada forma de crescimento os vetores têm uma tendência a se comportarem de forma diferente em comparação com as variáveis do vetor 4 (nenhuma forma de crescimento). No vetor 2 (crescimento inorgânico) as variáveis significantes foram: 1) nivel das vendas $(p$-value $=0,000)$, folga financeira $(p$-value $=0,026) \mathrm{e}$ grau de rentabilidade $(p$-value $=0,049)$. As duas primeiras apresentaram significância positiva e a ultima apresentou significância negativa. Portanto, quanto maiores os valores do nível de vendas e folga financeira, maior a probabilidade de ocorrência de crescimento inorgânico e quanto maior o valor do grau de rentabilidade, menor a probabilidade de ocorrência de crescimento inorgânico. Assim sendo não se rejeita as hipóteses 1, 3 e 6.

No vetor 3 (crescimento orgânico e inorgânico), a variável folga financeira apresentou significância positiva ( $p$-value $=0,002)$. Portanto, quanto maior o seu valor, maior a probabilidade de crescimento seja orgânico ou inorgânico.

\subsection{Comparativo do resultado com outras pesquisas}

Neste artigo a forma de Crescimento Orgânico - CO (em inglês Organic Growth) foi considerada como crescer construindo novas plantas, criando mercado pela própria firma crescimento do núcleo da empresa; a forma de Crescimento Inorgânico - CI (em inglês Inorganic Growth), por sua vez, foi considerada como crescer adquirindo plantas de outras firmas, com mercados já existentes, por meio de Aquisições de empresas. 
IDENTIFICAÇÃO DOS FATORES QUE INFLUENCIAM NA ESCOLHA DA FORMA DE INVESTIMENTO EM CRESCIMENTO (ORGÂNICO OU INORGÂNICO) DAS INDÚSTRIAS BRASILEIRAS

Quadro 3 - Modelo Logit Multinomial com erro padrão QML

\begin{tabular}{|c|c|c|c|c|c|c|}
\hline \multicolumn{7}{|c|}{ Forma_de_Cresc $=1$} \\
\hline Variáveis & Coeficiente & Std Err & $\boldsymbol{z}$ & $\boldsymbol{P}>\boldsymbol{z}$ & $\mathbf{9 5 \% \text { Conf }}$ & Interval \\
\hline Const & $--5,3453$ & 1,0197 & $-5,24$ & $<0,000 * * *$ & $-7,3440$ & $-3,3465$ \\
\hline Gr_Rent & 0,0228 & 0,0212 & 1,07 & 0,283 & $-0,0188$ & 0,0645 \\
\hline Ris_Neg & $-0,4311$ & 1,0584 & $-0,41$ & 0,684 & $-2,5056$ & 1,6433 \\
\hline Fol_Fin & 0,1601 & 0,1231 & 1,30 & 0,193 & $-0,8122$ & 0,4015 \\
\hline Ris_Fal & 0,0105 & 0,0266 & 0,40 & 0,692 & $-0,0416$ & 0,0626 \\
\hline Niv_End & $-0,1879$ & 0,2195 & $-0,86$ & 0,392 & $-0,6182$ & 0,2423 \\
\hline Log_Ven & 0,4034 & 0,4152 & 0,97 & 0,331 & $-0,4104$ & 1,2173 \\
\hline Log_At & 0,2396 & 0,4540 & 0,53 & 0,598 & $-0,6503$ & 1,1296 \\
\hline Q_Tobin & $-0,0042$ & 0,0037 & $-1,16$ & 0,247 & $-0,0115$ & 0,0029 \\
\hline
\end{tabular}

\begin{tabular}{|c|c|c|c|c|c|c|}
\hline \multicolumn{7}{|c|}{ Forma_de_Cresc $=\mathbf{2}$} \\
\hline Variáveis & Coeficiente & Std Err & $\boldsymbol{z}$ & $\boldsymbol{P}>\boldsymbol{z}$ & $\mathbf{9 5 \%}$ Conf & Interval \\
\hline Const & $-19,8593$ & 4,9133 & $-4,04$ & $<0,000 * * *$ & $-29,4893$ & -102293 \\
\hline Gr_Rent & $-0,1743$ & 0,0885 & $-1,97$ & $0,049 * * *$ & $-0,3479$ & $-0,0008$ \\
\hline Ris_Neg & 0,3396 & 2,6874 & 0,13 & 0,899 & $-4,9276$ & 5,6068 \\
\hline Fol_Fin & 0,4539 & 0,2037 & 2,23 & $0,026 * * *$ & 0,0546 & 0,8533 \\
\hline Ris_Fal & 0,0758 & 0,1336 & 0,57 & 0,570 & $-0,1861$ & 0,3379 \\
\hline Niv_End & $-0,0403$ & 0,2820 & $-0,14$ & 0,886 & $-0,5930$ & 0,5124 \\
\hline Log_Ven & 3,2119 & 0,8420 & 3,81 & $0,000 * * *$ & 1,5615 & 4,8623 \\
\hline Log_At & $-0,7621$ & 0,5736 & $-1,33$ & 0,184 & $-1,8864$ & 0,3621 \\
\hline Q_Tobin & 0,0380 & 0,0315 & $-1,20$ & 0,228 & $-0,0999$ & 0,0238 \\
\hline
\end{tabular}

\begin{tabular}{|c|c|c|c|c|c|c|}
\hline \multicolumn{7}{|c|}{ Forma_de_Cresc $=3$} \\
\hline Variáveis & Coeficiente & Std Err & $\boldsymbol{z}$ & $\boldsymbol{P}>\boldsymbol{z}$ & $\mathbf{9 5 \% \text { Conf }}$ & Interval \\
\hline Const & $-9,4155$ & 1,5862 & $-5,94$ & $<0,000 * * *$ & $-12,5246$ & $-6,3064$ \\
\hline Gr_Rent & $-0,0123$ & 0,0210 & $-0,58$ & 0,560 & $-0,0534$ & 0,0289 \\
\hline Ris_Neg & 0,7557 & 0,8365 & 0,90 & 0,366 & $-0,8837$ & 2,3953 \\
\hline Fol_Fin & 0,3755 & 0,1201 & 3,13 & $0,002^{* * *}$ & 0,1401 & 0,6110 \\
\hline Ris_Fal & 0,0457 & 0,0240 & $-1,90$ & $0,057 *$ & $-0,0929$ & 0,0014 \\
\hline Ni__End & $-0,3784$ & 0,3416 & $-1,11$ & 0,268 & $-1,0480$ & 0,2910 \\
\hline Log_Ven & 0,4244 & 0,5509 & 0,77 & 0,441 & $-0,6554$ & 1,5043 \\
\hline Log_At & 0,7459 & 0,5264 & 1,42 & 0,157 & $-2,2859$ & 1,7778 \\
\hline Q_Tobin & 0,0000 & 0,0025 & 0,30 & 0,765 & $-0,0042$ & 0,0058 \\
\hline
\end{tabular}

Forma_de_Cresc $=4$ (base autcome)

Fonte: Elaborado pelos autores

Conforme já evidenciado anteriormente, não encontramos nenhum estudo empírico, nacional ou internacional, que fizesse essa abordagem de verificar a existência de fatores que pudessem ser considerados determinantes para escolher a forma de investimento em crescimento, entre investimento em crescimento orgânico e investimento em crescimento inorgânico.

Com a escolha destas variáveis estudadas, como fatores determinantes para escolha da forma de investimento, não podemos afirmar de forma categórica que são as mais apropriadas. 


\section{CONCLUSÕES}

O conceito de crescimento de uma empresa é a representação da expansão de seu negócio obtido por meio do aumento da sua produção, melhorando a experiência, abrindo novos mercados e vendas, da inovação, lançamento de novos produtos, compromisso dos gestores e empregados das empresas, diversificação, entre outros. Essa expansão pode ocorrer por meio do crescimento orgânico ou inorgânico.

Partindo-se do pressuposto de expansão da firma, este artigo buscou responder ao seguinte problema de pesquisa: Existem fatores determinantes para a escolha entre investimento em crescimento orgânico e investimento em crescimento inorgânico, como alternativas de expansão das empresas?

As 8 hipóteses foram testadas em um primeiro momento por meio do modelo econométrico de Painel Binário de dados com a variável dependente binária utilizando o vetor (0) para crescimento orgânico e o vetor (1) para crescimento inorgânico. O modelo de efeito aleatorio foi mais adequado, com a variável nível de vendas, apresentando significância positiva. Entretanto, neste modelo, o regressando admitiu somente a preponderância de crescimento orgânico e inorgânico.

Em um segundo momento realizou-se a análise dos dados por meio do modelo econométrico Logit Multinomial com erro padrão robusto. Para vetor 2 (crescimento inorgânico) as variáveis nível das vendas e folga financeira apresentaram significância positiva, ou seja, quanto maiores o seus valores maior a probabilidade de crescimento inorgânico. A variável grau de rentabilidade apresentou significância negativa e, portanto, quanto maior seu valor menor a probabilidade de crescimento inorgânico.

No vetor 3 (crescimento orgânico e inorgânico), a variável folga financeira apresentou significância positiva. Logo, folga financeira implica em crescimento qualquer que seja sua natureza.

Considerando ambos os vetores pode-se afirmar que folga financeira aumenta probabilidade de crescimento, especialmente o inorgânico. Maior nível de vendas igualmente aumenta a probabilidade de investimento em crescimento inorgânico. E, finalmente, quanto maior a rentabilidade, menores as chances de crescimento inorgânico. Assim sendo não se rejeita as hipóteses 1,3 e 6 .

Este artigo surge como primeiro trabalho a estudar fatores que influenciam na escolha da forma de investimento em crescimento orgânico ou inorgânico nas indústrias brasileiras de capital aberto, portanto passiva de futuras discussões e novas pesquisas.

Portanto, como sugestão para estudos posteriores, com base nas limitações desta pesquisa, sugere-se ampliar a amostra, estendendo-a a todas as empresas de capital aberto do Brasil, podendo ampliar a pesquisa a nível internacional, fazendo comparações com outros países ou blocos comerciais.

Os resultados obtidos com esta pesquisa apontam que nem todas as variáveis exógenas do estudo apresentaram resultados relevantes, indicando que não são fatores para a escolha da forma de investimento em crescimento.

A escolha das variáveis se deu com base na tentativa de amarrar com a fundamentação teórica, assim com a escolha destas variáveis estudadas, como fatores determinantes para escolha da forma de investimento, não podemos afirmar de forma categórica que são as mais apropriadas.

Uma das limitações deste estudo foi o fato de considerarmos o "anúncio" de investimento em crescimento orgânico ou inorgânico, sem levar em consideração se os reflexos financeiros dessas escolhas foram positivos ou negativos em relação ao retorno. Entende-se que essa possibilidade pode abrir novas oportunidades para futuras investigações. 
Considerou-se ainda como limitação do estudo, a questão relacionada às fontes dos investimentos, ou seja, não foram abordadas as origens dos recursos (próprios ou de terceiros) anunciados como investimento em crescimento orgânico ou inorgânico. Isto poderá acarretar diferenças nos resultados de pesquisas futuras, caso esta abordagem venha a ser realizada.

Os resultados desta pesquisa em termos gerais contribuem para elucidar a questão proposta inicialmente, oferecendo evidências empíricas da relação a alguns fatores que são determinantes para a escolha da forma de investimento em crescimento orgânico ou inorgânico nas indústrias brasileiras no período analisado.

\section{REFERÊNCIAS}

ANDRADE, M.M. Como preparar trabalhos para cursos de pós-graduação: noções práticas. 5. ed. São Paulo: Atlas, 2002.

BALAKRISHNAN S.; FOX, I. Asset specificity, firm heterogeneity and capital structure. Strategic Management Journal, v. 14, n. 1, p. 3-16. 1993. http://dx.doi.org/10.1002/smj.4250140103

BARNEY, J.B. Firm resources and sustained competitive advantage. Journal of Management, v. 17, n. 1, p. 99-120, 1991. http://dx.doi.org/10.1177/014920639101700108

BESANKO, David et al. Economics of strategy. 3rd ed. EUA: John Wiley \& Sons, 2004.

BORODZICZ, E. P. Risk, crisis and management. New York: John Wiley \& Sons. 2005

BREALEY A.R.; MYERS C.S. Principles of Corporate Finance. 7. ${ }^{a}$ ed. McGraw-Hill, 2005.

CAPRON, L.; PISTRE, N. When do acquirers earn abnormal returns? Strategic Management Journal, v.23, n.9, p.781-794, 2002. http://dx.doi.org/10.1002/smj.262

CHANDLER, A. Scale and scope: the dynamics of Industrial capitalism. Harvard: Belknap, 1990.

COASE, R. H. The nature of the firm. Economica, v. 4, n. 16, p. 386-405. 1937. http://dx.doi.org/10.1111/j.1468-0335.1937.tb00002.x

COPELAND, T.E.; WESTON, F.J. Financial theory and corporate policy. Reimpressão da 3a. edição. Nova Iorque: Addison Wesley. 1992.

CORREA, C.A; BASSO, L.F.C.; NAKAMURA, W.T. A etrutura de capital das maiores empresas brasileiras: Análise empírica das teorias de Pecking Order e Trade-Off, usando Panel Data. In: Enanpad, 2007. Anais... Rio de Janeiro, 2007.

FAMÁ, R.; PEROBELLI, F.F. Fatores determinantes da estrutura de capital: Aplicação a empresas de capital aberto no Brasil. In: Encontro Brasileiro de Finanças. São Paulo, 1., 2001. Anais ..., 2001

GIL, A.C. Métodos e técnicas de pesquisa social. 5. ed. São Paulo: Atlas, 1999.

GRINBLATT, Mark; TITMAN, Sheridan. Mercados financeiros e estratégia corporativa. 2. ${ }^{\text {a }}$ Ed. Porto Alegre: Bookman, 2005. 
GUJARATI, D.N. Econometria básica. Tradução de Maria Jose Cyhlar Monteiro. 2. ${ }^{\text {a }}$ Ed. Rio de Janeiro, Elsevier, 2006.

HART, O. An economist's perspective on the theory of the firm, Columbia Law Review 1757, 1989, também em Buckley, P.J. e J. Michie (eds), Firms, Organizations and Contracts, Oxford University Press, Oxford, 1996.

HAUSMAN, J.A. Testes da especificação na econometria. V. 46, n. 6, p. 1251-1271. Novembro, 1978

HENNART, J.F.; PARK, Y.R. Greenfield vs. acquisition: The strategy of Japanese investors in the United States, Management Science, September, p.1054-1070. 1993. http://dx.doi.org/10.1287/mnsc.39.9.1054

HESS, EDWARD D. The road to organic growth. How great companies consistently grow marketshare from within. New York, McGraw-Hill, 2007.

IYER, D.N.; MILLER, K.D. Performance feedback, slack, and the timing of acquisitions. Academy os Management Journal. v. 51, n. 4, p. 808-822, 2008.

JAAFARI, A. Management of risks, uncertainties and opportunities on projects: time for a fundamental shift. International Journal of Project Management. v. 19, p. 89-101. 2001. http://dx.doi.org/10.1016/S0263-7863(99)00047-2

JOYCE, W.; NOHRIA, N.; ROBERSON, B. What really works: the $4+2$ formula for sustained business success. Harper Business, 2003.

KAYO, E.K. A estrutura de capital e o risco das empresas tangível e intangível-intensivas: uma contribuição ao estudo de valoração das empresas. Tese (Doutorado), FEA/USP, Faculdade de Economia, Administração e Contabilidade, USP, 2002.

KAYO, E.K.; PATROCINIO, M.R.; MARTIN, D.M.L. Intangibilidade e criação de valor em aquisições: o papel moderador do endividamento. In: Encontro da Anpad, 2008, Rio de Janeiro. Anais..., 2008.

KAZANJIAN, R.K.; HESS, E.G.; DRAZIN, R. The search for organic growth. Cambridge University Press. 2006

LELAND, Hayne; PYLE, David. Information asymmetries, financial structure and financial intermediation. The Journal of Finance, v.32, n.2, may, 1977.

MARCH, J.G.; SHAPIRA, Z. Managerial perspectives on risk and risk taking. Management Science, v. 33, p. 1404-1418. 1987. http://dx.doi.org/10.1287/mnsc.33.11.1404

PENROSE, Edith. The theory of the growth of the firm. United States: Oxford University. New York, 1959.

PEREIRA, J. Silva. Análise financeira das empresas. 10. ${ }^{a}$ edição. São Paulo, Atlas, 2010.

RICHIERI, Flávio L. Capital intelectual e a criação de valor nas empresas brasileiras. Dissertação de Mestrado. Universidade Presbiteriana Mackenzie, 2007. 
IDENTIFICAÇÃO DOS FATORES QUE INFLUENCIAM NA ESCOLHA DA FORMA DE INVESTIMENTO EM CRESCIMENTO (ORGÂNICO OU INORGÂNICO) DAS INDÚSTRIAS BRASILEIRAS

SEKARAN, U. Research methods for business: a skill-building approach. 2 nd ed. New York: John Wiley, 1992. Cap.3 e 4 (p.62-113)

SOLOW. R.M. Economic History and economics. AEA Papers and Proceedings, May 1985; vol.75, n.2. 1985.

STULZ, R. Managerial discretion and optimal financing policies. Journal of Financial Economics, v.26, 3-27, 1990. http://dx.doi.org/10.1016/0304-405X(90)90011-N

TITMAN, S.; WESSELS, R. The determinants of capital structure choice. Journal of Finance, v. 48, n. 3, June 1988.

VERBEEK, Marno. A guide to modern econometrics. $1^{\text {a }}$ ed. Reimpressa com correções. Buffins Lane: John Wiley \& Sons, 2001.

VILLALONGA, B. Intangible resources, Tobin's q, and sustainability of performance differences. Journal of Economic Behavior \& Organization, v.54, n.2, p.205-230, 2004. http://dx.doi.org/10.1016/j.jebo.2003.07.001

VILLALONGA, B.; McGAHAN. The choice among acquisitions, alliances, and divestitures. Strategic Management Journal, v.26, n.13, 2005. http://dx.doi.org/10.1002/smj.493

WILK, E.O. O uso da abordagem resource-based view na formulação de estratégias: ocaso do setor vitivinícula da Serra Gaúcha. In: Congresso ENANPAD, 2000. Anais..., 2000.

WILLIAMSON, O.E. Markets and hierarchies: Analysis and antitrust implications. New York: Free Press. 1975.

WILLIAMSON, O.E. The economic institutions of capitalism: Firms, markets, relational contracting. New York: The Free Press, 1985. 